\title{
Bulk Growth and Characterization of D-(-)-Alanine Single Crystals
}

\author{
Kalimuthu Moovendaran, Subramanian Natarajan ${ }^{*}$ \\ School of Physics, Madurai Kamaraj University, Madurai, India.

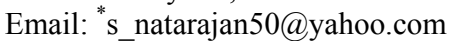 \\ Received July $2^{\text {nd }}, 2013$; revised August $2^{\text {nd }}, 2013$; accepted August $9^{\text {th }}, 2013$ \\ Copyright (C) 2013 Kalimuthu Moovendaran, Subramanian Natarajan. This is an open access article distributed under the Creative \\ Commons Attribution License, which permits unrestricted use, distribution, and reproduction in any medium, provided the original \\ work is properly cited.
}

\begin{abstract}
Single crystal of D-(-)-alanine (DALA), a non-linear optical material from the amino acid family was grown using a home-made crystal growth setup. The crystals of DALA were also grown by slow evaporation solution technique (SEST). The grown crystals were characterized by using single crystal X-ray diffraction, high resolution X-ray diffraction (HRXRD) and UV-vis-NIR and CD spectroscopy. Measurements of Vicker's microhardness, laser damage threshold (LDT) value and second harmonic generation (SHG) efficiency are also reported. Thermal and dielectric studies were also carried out.
\end{abstract}

Keywords: D-(-)-Alanine; NLO Material; MKN Setup; HRXRD; CD; SHG

\section{Introduction}

The search for new materials with high optical nonlinearities is an important field of research due to their practical applications in harmonic generation, amplitude and phase modulation, switching and other signal processing devices. Alanine is the simplest amino acid with an asymmetric carbon atom. L-Alanine and DL-Alanine are the usually available forms of alanine. D-Amino acids do not occur in nature and are usually synthesized by manufacturers. The crystals of L-alanine [1] and DL-alanine [2] were grown by slow evaporation method, characterized and their NLO property established. Recently, the spectral characterization, second harmonic generation (SHG) and hyper Raleigh scattering studies of D-(-)-alanine were reported from our laboratory [3]. The bulk growth of the single crystal of D-(-)-alanine has not been reported till date. In the present investigation, a bulk single crystal of D-(-)-alanine (DALA) was grown using a home-made crystal growth setup. The crystals of DALA were also grown by slow evaporation solution technique (SEST). The crystals were characterized by using XRD, HRXRD and spectroscopic techniques. Several useful properties of the crystal, such as microhardness, LDT and SHG were evaluated. Thermo gravimetric and differential thermal analysis curves were recorded and interpreted. Measurements of dielectric constant and loss

${ }^{*}$ Corresponding author. were also made.

\section{Crystal Growth Experiments}

\subsection{Growth of DALA Crystals Using Slow Evaporation Solution Technique (SEST)}

D-Alanine $\left(\mathrm{C}_{3} \mathrm{H}_{7} \mathrm{NO}_{2}\right)$ is a white crystalline powder with a molecular weight of 89.09 . Commercially available D-alanine (M/s. Loba Chemie Pvt. Ltd., purity: 99\%) was further purified by recrystallising it once using water as the solvent. A saturated solution of D-alanine was prepared and allowed to evaporate at a room temperature $\left(30^{\circ} \mathrm{C}\right)$. Transparent colourless crystals of size: $10 \times 5 \times 3$ $\mathrm{mm}$ were obtained within a period of one week.

\subsection{Growth of a Bulk Crystal Using a Home Made Setup}

A new setup was designed in our laboratory to conduct several crystal growth experiments simultaneously [4]. The home made setup (called as MKN setup) is made up of two large tanks and it consists of several ampoules, dimmerstat, temperature controller, heating coil and a thermometer. Constant temperature and uniform temperature gradient were maintained, to increase the growth rate and quality of the crystal grown. The details of this setup has been given elsewhere [4,5]. A suitable seed crystal was chosen from the crystals obtained by the 
SEST experiment and mounted for the crystal growth along the [020] direction. An aqueous saturated solution was prepared and transferred to the growth ampoule. A growth run for 20 days resulted in a crystal of sizes: 15 $\mathrm{mm}$ diameter and $45 \mathrm{~mm}$ length, the average growth rate being about $2 \mathrm{~mm}$ per day. The photograph of the grown crystal of DALA is shown in Figure 1.

\section{Characterization}

The grown crystal was subjected to single crystal X-ray diffraction studies using an Enraf Nonius CAD-4/MACH 3 diffractometer, with $\mathrm{MoK} \alpha$ radiation $(0.71073 \AA)$. The accurate cell parameters of the grown crystals at room temperature $\left(25^{\circ} \mathrm{C}\right)$ were obtained from the least-squares refinement of the setting angles of 25 reflections. To study the crystalline perfection of the grown crystals, a multicrystal X-ray diffractometer developed at the National Physical Laboratory, New Delhi [6] has been used for recording the high-resolution diffraction curves (DCs) for $\left(\begin{array}{lll}0 & 2 & 0\end{array}\right)$ diffracting plane in symmetrical Bragg geometry. The description of the above instrument has already been given elsewhere [7]. The transmittance of the grown crystal (for a crystal of thickness: $2 \mathrm{~mm}$ ) was measured using a Perkin-Elmer Lambda-35 spectrophotometer in the wavelength range of $200-1100 \mathrm{~nm}$ with a slit of width $2 \mathrm{~nm}$ and scan speed of $240 \mathrm{~nm} / \mathrm{min}$. CD spectra were recorded in the wavelength range of 150 $400 \mathrm{~nm}$ at room temperature, using a JASCO J-180 spectropolarimeter. The aqueous solution $(3 \mathrm{mM})$ of the sample was kept in a quartz cuvette of path length $0.1 \mathrm{~cm}$. Microhardness measurements were made on the cut and polished plate of the crystal using a Shimadzu Microhardness Tester (Model No. HMV2T) with a diamond indenter. The well-polished crystal was mounted on the platform of the microhardness tester and loads of different magnitudes were applied over a fixed interval of time (8 s). The laser damage threshold (LDT) measurement has been carried out for the grown crystal using a Q-swtiched Nd: YAG laser $(\lambda=1064 \mathrm{~nm})$. The second

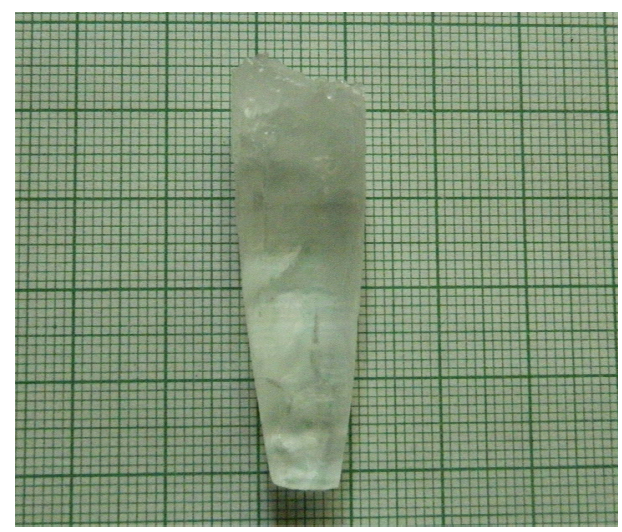

Figure 1. Photograph of the grown crystal. harmonic generation behaviour of the powdered materials was tested using the Kurtz and Perry method [8], making use of the laser source described above. Simultaneous thermogravimetry (TG) and differential thermal analyses (DTA) of powdered samples were performed in the temperature range of $25^{\circ} \mathrm{C}$ to $900^{\circ} \mathrm{C}$, using a Netzsch STA $409 \mathrm{PC} / \mathrm{PG}$ thermal analyzer at a heating rate of $10^{\circ} \mathrm{C} / \mathrm{min}$. An $\mathrm{Al}_{2} \mathrm{O}_{3}$ (alumina) crucible was used and it served as a reference for the sample. Dielectric constant and loss measurements were made using a HIOKI $352-50 \mathrm{LCR}$ meter in the frequency range of $100 \mathrm{~Hz}-1$ $\mathrm{MHz}$. Crystals of reasonable dimensions were cut from the bulk crystal and polished using the solvent (water). A silver coating was applied on the opposite sides of the crystal and placed between two copper electrodes and thus a parallel plate capacitor was formed.

\section{Results and Discussion}

\subsection{Single Crystal X-Ray Diffraction}

From the single crystal X-ray diffraction studies, the lattice parameters were determined as: $\mathrm{a}=6.043(3), \mathrm{b}=$ $12.337(5)$ and $c=5.784(3) \AA$. These values are in agreement with those reported by Sullivan et al. [9]. It is already known that this compound crystallizes in the orthorhombic system with the space group $\mathrm{P} 22_{1} 2_{1} 2_{1}$. The density of the single crystals of DALA was determined as $1.37(2) \mathrm{gm} / \mathrm{cm}^{3}$ using the floatation method. The melting point was found out as $290(2)^{\circ} \mathrm{C}$.

\subsection{High-Resolution X-Ray Diffraction Analysis}

The high-resolution diffraction curve (DC, Figure 2) was recorded for the DALA single crystal using ( $\left.\begin{array}{lll}0 & 2 & 0\end{array}\right)$ diffracting planes in symmetrical Bragg geometry by employing the multicrystal X-ray diffractometer described above with $\mathrm{MoK} \alpha_{1}$ radiation. On deconvolution of the diffraction curve, it is clear that the curve contains an additional peak which is 105 arc s away from the main peak. The additional peak corresponds to an internal structural low angle grain boundary. For better understanding, the schematic of a structural grain boundary is given in the inset of Figure 2(a). As seen in the inset, two regions of the crystal are misoriented by a finite angle $\alpha$ also known as tilt angle. Tilt angle may be defined as the misorientation angle between the two crystalline regions on both sides of the structural grain boundary. The two regions may be perfect. If the value of $\alpha$ is $\leq 1$ arc min, it is considered as very low angle boundary. If $\alpha$ $>1$ arc min but less than a deg, it is considered as low angle boundary. The tilt angle for the very low angle boundary is 105 arc $\mathrm{s}$ with respect to the main crystal block. The full width at half maximum (FWHM) of the main peak and the boundary are respectively 23 and 30 arc s. The low FWHM values of main peak and the very 


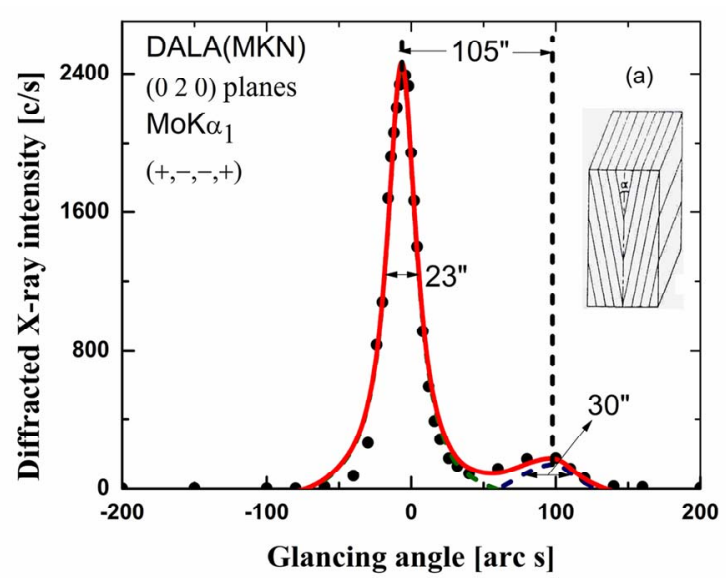

Figure 2. High resolution DC for the crystal.

low angle boundary indicate that the crystalline perfection of the specimen is reasonably good. It may be mentioned here that such minute defects could be detected with well resolved peaks in the diffraction curve only because of the high-resolution of the diffractometer, characterized by very low values of wavelength spread i.e. $\Delta \lambda / \lambda$ and horizontal divergence for the exploring or incident beam, which are respectively around $10^{-5}$ and much less than 3 arc s of the multicrystal X-ray diffractometer used in the present studies.

\subsection{UV-vis-NIR and CD Spectroscopic Studies}

From the UV-vis-NIR transmission spectrum (Figure 3), it is seen that the compound has no absorption in the region between $229 \mathrm{~nm}$ and $1100 \mathrm{~nm}$. The optical transmittance of the crystals grown using the MKN setup is $52 \%$, which is $6 \%$ higher than that for the crystal grown by SEST. The reason for the higher transparency of the crystal grown using the MKN setup may be the absence of inclusions, which reduces absorption of the UV-visNIR radiation. The CD spectrum of DALA unambiguously confirms its chiral purity and thus enables its characterization as D-(-)-alanine (Figure 4). From the negative Cotton effect observed, it can be concluded that the compound DALA is D-(-)-alanine.

\subsection{Microhardness Studies}

Microhardness is an important parameter which helps to know about the mechanical properties of materials such as fracture behavior, yield strength, brittleness index and temperature of cracking. Figure 5 shows the plot between the hardness value $(\mathrm{Hv})$ and load P. From the results, it is observed that the hardness value increases with increase of load up to $50 \mathrm{~g}$ and with a further increase in applied load, cracks have been observed on the crystal surface due to the release of internal stress generated locally by indentation. It is also observed that the me-

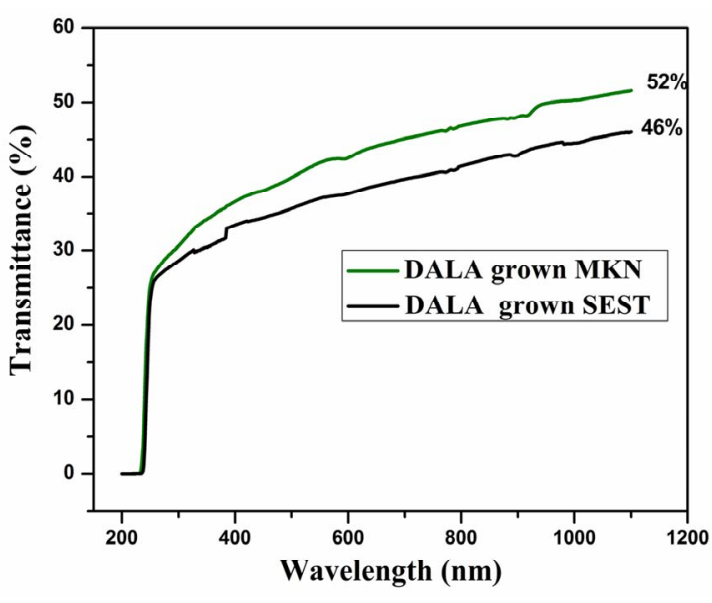

Figure 3. Transmission spectrum of DALA.

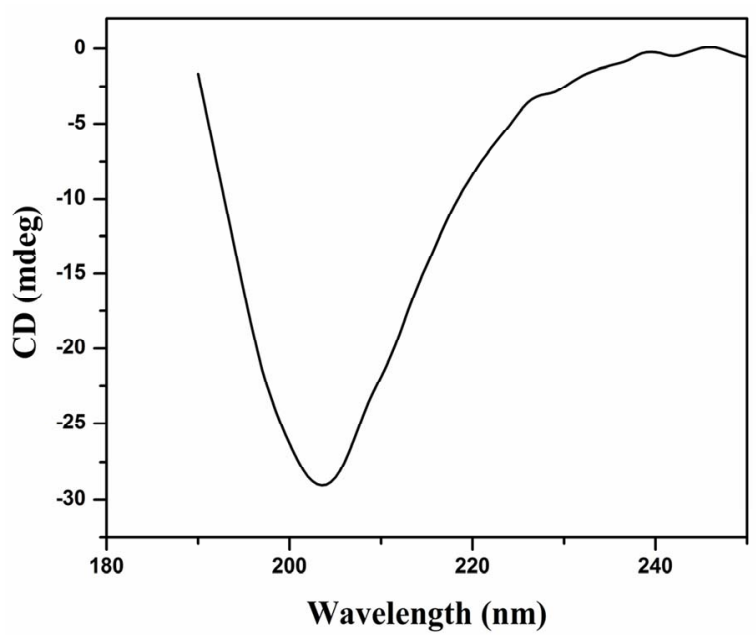

Figure 4. CD spectra of D-(-)-alanine.

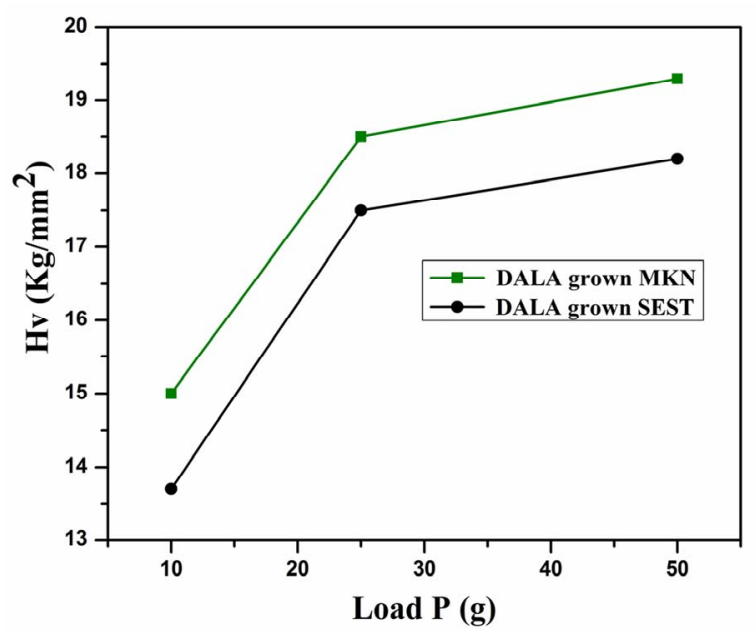

Figure 5. Variation of Vickers hardness number with applied load.

chanical stability of the DALA crystal grown in the MKN setup is higher compared to the crystals grown by 
SEST. A plot obtained between $\log (\mathrm{P})$ and $\log (\mathrm{d})$ gives a straight line (Figure 6). The relation connecting the applied load (P) and diagonal length $(\mathrm{d})$ of the indenter is given by the Meyer's law: $\mathrm{P}=\mathrm{ad}^{\mathrm{n}}$, where, $\mathrm{n}$ is the Meyer's index or work hardening coefficient and a, a constant of proportionality. The work hardening coefficient value (n) of the crystals grown using SEST and the MKN setup were determined by the least-squares fit method and found to have a values of 1.75 and 1.68, respectively. Onitsch [10] has pointed out that $\mathrm{n}$ lies between 1.0 and 1.6 for moderately hard materials and it is more than 1.6 for soft materials. Hence, it is concluded that DALA single crystal is a soft material.

\subsection{Laser Damage Threshold (LDT) Measurements}

The laser damage threshold (LDT) measurement has been carried out for the grown crystal using a Q-swtiched $\mathrm{Nd}$ : YAG laser $(\lambda=1064 \mathrm{~nm})$ with pulse width of $10 \mathrm{~ns}$, operated at the repetition rate of $10 \mathrm{~Hz}$. The laser beam (1 mm diameter) was focused on the sample using a lens with focal length of $20 \mathrm{~cm}$. The spot size of the focused beam on the sample was $0.3 \mathrm{~mm}$. The measured LDT values of DALA single crystals grown using SEST and the MKN setup are $6.8 \mathrm{GW} / \mathrm{cm}^{2}$ and $7.5 \mathrm{GW} / \mathrm{cm}^{2}$, respectively. It is observed that the crystal grown in the MKN setup has slightly higher LDT value than that of the crystal grown by SEST.

\subsection{Second Harmonic Generation (SHG) Conversion Efficiency}

Quantitative measurement of the SHG conversion efficiency of the sample was made using the powder technique developed by Kurtz and Perry. The output from the sample was monochromated to collect only the second

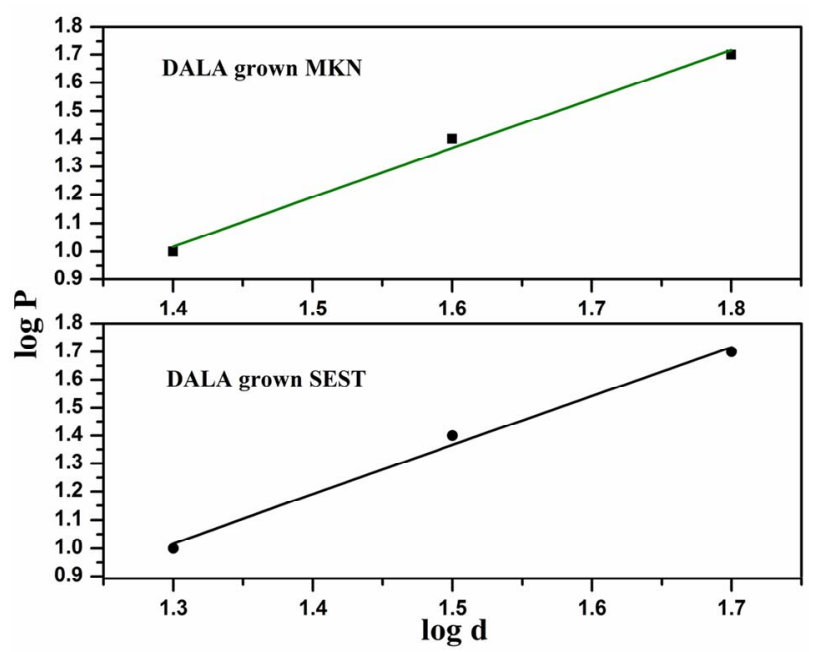

Figure 6. Plot of $\log d$ vs $\log P$. harmonic $(\lambda=532 \mathrm{~nm})$, eliminating the fundamental and the intensity was measured using a photomultiplier tube. A SHG signal of $3 \mathrm{mV}$ for the crystal was obtained for an input energy of $3.9 \mathrm{~mJ} /$ pulse. The standard KDP crystal gave a SHG signal of $13 \mathrm{mV}$ for the same input energy. It is seen that the SHG efficiency of the sample crystal is about $23 \%$ of that of the standard KDP crystals.

\subsection{Thermal Studies}

In the TG-DTA (Figure 7), an endothermic peak observed at $290^{\circ} \mathrm{C}$ in DALA corresponds to the melting point of the material. It is seen from the TG plot that the melting and decomposition of the compound occur simultaneously. Hence, this compound may be useful for its applications upto its melting point. The exothermic peak observed at $466^{\circ} \mathrm{C}$ is due to the phase transition expected to happen in the carbon residue.

\subsection{Dielectric Studies}

The dielectric constant and dielectric loss of the DALA crystal were studied as a function of frequency, at different temperatures $\left(30^{\circ} \mathrm{C}, 40^{\circ} \mathrm{C}, 50^{\circ} \mathrm{C}\right.$ and $\left.60^{\circ} \mathrm{C}\right)$. However, the variation of dielectric constant and the dielectric loss with frequency only at the room temperature $\left(30^{\circ} \mathrm{C}\right)$ are shown in Figure 8. It is observed from the plots, that both the dielectric constant $\left(\varepsilon^{\prime}\right)$ and the dielectric loss $\left(\varepsilon^{\prime \prime}\right)$ are decreasing rapidly and get saturated at high frequencies. A comparison of the dielectric response of the crystals suggests that the dielectric constant and dielectric loss of the crystals grown using the MKN setup are less compared to that for the crystal grown by SEST. The low values of dielectric loss at high frequencies suggest that the crystal grown using the MKN setup possesses enhanced optical quality with low density of defects [11].

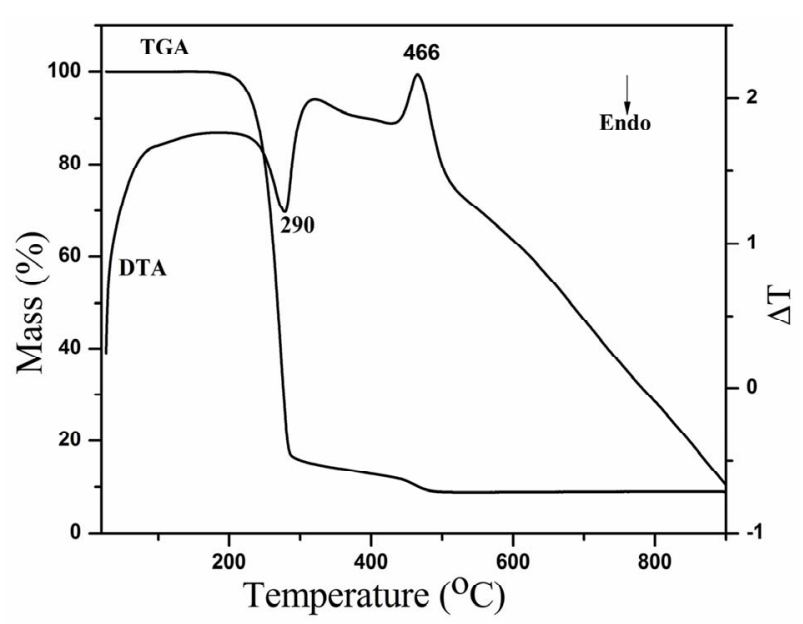

Figure 7. TG-DTA plots of DALA. 


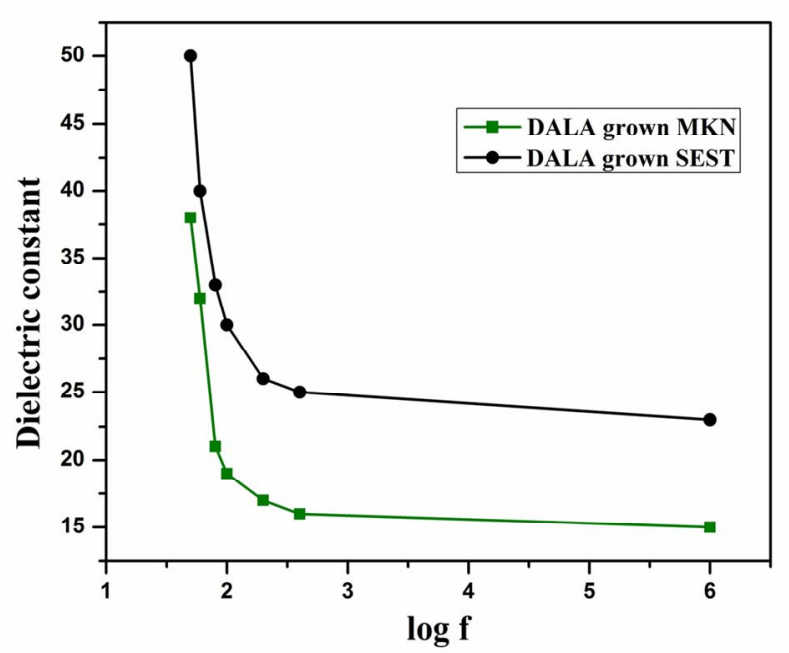

(a)

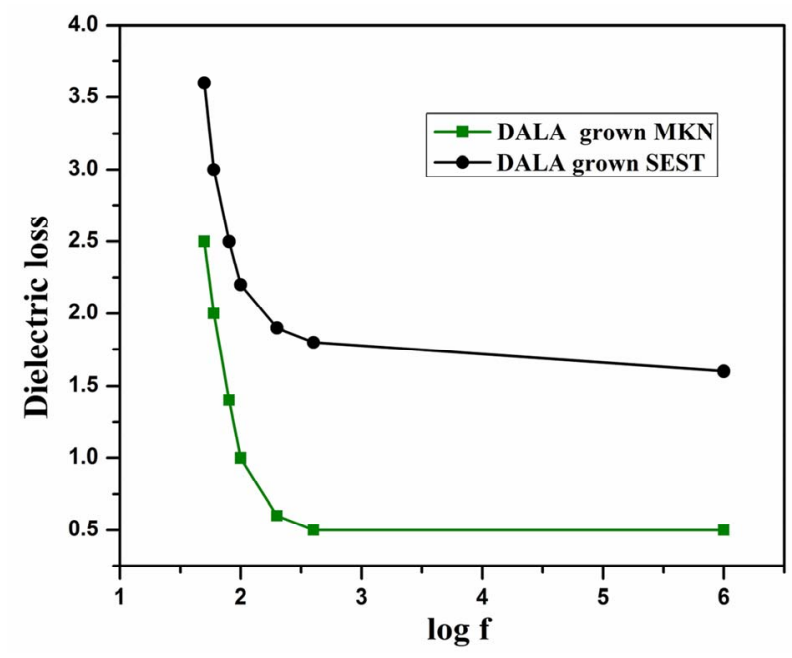

(b)

Figure 8. (a) The variation of dielectric constant with frequency; (b) The variation of dielectric loss with frequency.

\section{Conclusion}

Bulk NLO crystal of D-(-)-alanine was grown using a home-made (MKN) crystal growth setup. The optical transparency, the values of LDT, microhardness, dielectric constant and dielectric loss are found to be better for the crystal grown in the MKN setup compared to that grown by SEST. The bulk crystal of DALA also possesses a high melting point and reasonably good crystalline perfection. The value of the SHG conversion efficiency of the crystal was found to be about $23 \%$ of that of the standard KDP crystal. Hence, the crystal of D$(-)$-alanine grown using the MKN setup may have some applications as a NLO material.

\section{Acknowledgements}

The authors are thankful to Dr. G. Bhagavannarayana,
National Physical Laboratory, New Delhi, for the help in recording the HRXRD curve. SN thanks the Council of Scientific and Industrial Research, New Delhi for the financial support under the Emeritus Scientist Scheme.

\section{REFERENCES}

[1] N. Vijayan, S. Rajesekaran, G. Bhagavannarayana, R. Ramesh Babu, R. Gopalakrishnan, M. Palanichamy and P. Ramasamy, "Growth and Characterization of Nonlinear Optical Amino Acid Single Crystal: L-Alanine," Crystal Growth \& Design, Vol. 6, No. 11, 2006, pp. 2441-2445. http://dx.doi.org/10.1021/cg049594y

[2] S. A. Martin Britto Dhas and S. Natarajan, "Growth and Characterization of DL-Alanine-A New NLO Material from the Amino Acid Family," Materials Letters, Vol. 62, No. 17-18, 2008, pp. 2633-2636. http://dx.doi.org/10.1016/j.matlet.2008.01.034

[3] K. Moovendaran, S. A. Martin Britto Dhas and S. Natarajan, "Spectral Characterization of a Non-Centrosymmetric Organic Compound: D-(-)-Alanine," Spectrochimica Acta Part A, Vol. 112, No. 13, 2013, pp. 326-330. http://dx.doi.org/10.1016/j.saa.2013.04.025

[4] K. Moovendaran, J. Kalyana Sundar and S. Natarajan, "Simultaneous Growth of Several Materials Using a Single Experimental Setup," Journal of Crystal Growth, Vol. 334, No. 1, 2011, pp. 1-3. http://dx.doi.org/10.1016/j.jcrysgro.2011.08.026

[5] K. Moovendaran and S. Natarajan, "Unidirectional Growth and Characterization of L-Tartaric Acid Single Crystals," Journal of Applied Crystallography, Vol. 46, No. 4, 2013, pp. 993-998.

http://dx.doi.org/10.1107/S0021889813011680

[6] K. Lal and G. Bhagavannarayana, "A High-Resolution Diffuse X-Ray Scattering Study of Defects in Dislocation-Free Silicon Crystals Grown by the Float-Zone Method and Comparison with Czochralski-Grown Crystals," Journal of Applied Crystallography, Vol. 22, No. 3, 1989, pp. 209-215. http://dx.doi.org/10.1107/S0021889888014062

[7] S. A. Martin Britto Dhas, M. Suresh, G. Bhagavannarayana and S. Natarajan, "Growth and Characterization of L-Tartaric Acid, an NLO Material," Journal of Crystal Growth, Vol. 309, No. 1, 2007, pp. 48-52. http://dx.doi.org/10.1016/j.jcrysgro.2007.09.008

[8] S. K. Kurtz and T. T. Perry, "A Powder Technique for the Evaluation of Nonlinear Optical Materials," Journal of Applied Physics, Vol. 39, No. 8, 1968, pp. 3798-3813. http://dx.doi.org/10.1063/1.1656857

[9] R. Sullivan, M. Pyda, J. Pak, B. Wunderlich, J. R. Thompson, R. Pagni, H. Pan, C. Barnes, P. Schwerdtfeger and R. Compton, "Search for Electroweak Interactions in Amino Acid Crystals. II. The Salam Hypothesis," The Journal of Physical Chemistry A, Vol. 107, No. 34, 2003, pp. 66746680. http://dx.doi.org/10.1021/jp0225673

[10] E. M. Onitsch, "The Present Status of Testing the Hardness of Materials," Mikroskopie, Vol. 95, No. 15, 1956, pp. 12-14. 
[11] C. Balarew and R. Duhlew, "Application of the Hard and Soft Acids and Bases Concept to Explain Ligand Coordination in Double Salt Structures," Journal of Solid State
Chemistry, Vol. 55, No. 1, 1984, pp. 1-6.

http://dx.doi.org/10.1016/0022-4596(84)90240-8 